\title{
Evaluación del crecimiento y productividad del tomate (Lycopersicon esculentum Mill) bajo cultivo protegido en tres localidades de Costa Rica
}

\author{
Carlos Ramírez Vargas' \\ James Nienhuis ${ }^{2}$
}

Fecha de recepción: 31 de enero del 201 I Fecha de aprobación: 3 de setiembre del 2011

Ramírez, C; Nienhuis, J. Evaluación del crecimiento y productividad del tomate (Lycopersicon esculentum Mill) bajo cultivo protegido en tres localidades de Costa Rica. Tecnología en Marcha. Vol. 25, N I. Enero-Marzo 2012 Pág 3-15 


\section{Palabras clave}

Horticultura, tomate, cultivo protegido, componentes principales, hábito de crecimiento, genotipo, ambiente.

\section{Resumen}

Mediante un experimento, se evaluó el crecimiento y la productividad de tres genotipos de tomate en tres localidades de Costa Rica con un sistema de cultivo protegido (San Blas de Carrillo en la provincia de Guanacaste; Santa Clara de San Carlos en la Provincia de Alajuela y también en Zapote de Zarcero en la provincia de Alajuela). Se evaluaron 19 variables fisiológicas de crecimiento y productividad para nueve tratamientos o interacciones.

A los datos obtenidos se les hizo un análisis de componentes principales y como resultado de este análisis se seleccionaron los dos primeros componentes que explican más del $70 \%$ de la variabilidad total, también se determinó que el componente principal I explica el crecimiento del cultivo, y su variable representativa fue el peso seco de tallos; mientras que el componente 2 representa el rendimiento, y su variable representativa fue el peso seco de frutos.

Se encontró también interacción de los genotipos con los ambientes pese a ser cultivados en invernadero, lo que demuestra una adaptación específica de los genotipos a las localidades que mostraron datos climáticos diferentes. La productividad de los genotipos también fue influenciada por los ambientes, sin embargo, no superaron los diez kilogramos de fruta fresca por planta.

\section{Key words}

Horticulture, tomato, protected culture, principal components, grow habit, genotype, environment.

\begin{abstract}
An experiment that involved the assessment of growth and productivity of tomato genotypes in three locations in Costa Rica in protected cultivation system was carried out. Communities were San Blas de Carrillo, Guanacaste Province; Santa Clara, San Carlos and Zapote, Alfaro Ruiz in the province of Alajuela. 19 physiological variables were evaluated for growth and productivity for 9 treatments or interactions.

The data obtained were made to a principal components analysis. As a result of this analysis, the first two components explained $70 \%$ of the total variability; it was determined that the principal component I explains the growth of the crop and variable representative was dry stem weight; component 2 represents the proxy performance and was the dry weight of fruit.

It was also found interaction of genotypes with environments despite being grown in the greenhouse, demonstrating a specific adaptation of genotypes to the locations that showed different climate data. The productivity of the genotypes was also influenced by the environments, but did not exceed $10 \mathrm{kgs}$ of fresh fruit per plant.
\end{abstract}




\section{Introducción}

La horticultura en Costa Rica se lleva a cabo principalmente en el Valle Central a campo abierto con uso intensivo del suelo, lo que conlleva una serie de problemas acumulativos como consecuencia del cultivo a lo largo de todo el año, circunstancia que puede verse como ventajosa en el trópico. Sin embargo, la presión de plagas y enfermedades se incrementa conforme el agricultor siembra en una misma zona un único cultivo, así como con un control de plagas y enfermedades centrado en el uso de plaguicidas químicos.

Otras formas de producción de hortalizas, como los sistemas de cultivo protegido, suponen una ventaja comparativa respecto al campo abierto, ya que el sistema favorece la alta productividad y el control de plagas y enfermedades, donde se puede hacer uso de infraestructura y equipo con el fin de tener un ambiente controlado. En el trópico, la implementación de esta tecnología tendría que solucionar problemas como las altas precipitaciones, alta temperatura y humedad relativa, al igual que la protección ante las plagas y enfermedades.

Una de las hortalizas más importantes en Costa Rica es el tomate. Para la temporada 2006-2007, se sembraron un total de 945,7 hectáreas, donde la mayor concentración se encontró en la región central occidental (provincias de Heredia y Alajuela) con 585 hectáreas, y únicamente 20 bajo sistema de cultivo protegido (Ministerio de Agricultura y Ganadería, citado en El Occidente, Alternativa en Información, año 15, Número 124, Mayo 2009; Consejo Nacional de Producción, 2008); en Centroamérica, la producción la encabezan Guatemala, Honduras y Costa Rica con $187.000,53.000$ y 50.000 toneladas métricas, respectivamente, durante ese mismo año (Benton-Jones, 2008).

La limitación de zonas con clima y suelos aptos para la producción de esta hortaliza de origen mesoamericano nos plantea el reto de implementar sistemas de producción donde se pueda tener un mejor control del medio edáfico-climático (Castilla, 2004), y así plantear la necesidad de investigación y validación de sistemas de cultivo protegido en un ambiente tropical como el de Costa Rica. Se debe investigar su adaptación al sistema en regiones no tradicionales para tomate, por medio de una evaluación, en primera instancia, de genotipos que presenten adaptabilidad a diferentes regiones, con características que permitan su manejo en búsqueda de altas productividades.

El objetivo del siguiente trabajo fue determinar la interacción entre tres cultivares de tomate con diferente hábito de crecimiento y en tres localidades diferentes de Costa Rica bajo el sistema de cultivo protegido, mediante la evaluación de su crecimiento y productividad.

\section{Materiales y métodos}

\section{Descripción general}

Se sembraron, en tres localidades de Costa Rica, tres híbridos de tomate con diferente hábito de crecimiento dentro de invernaderos bajo sistema hidropónico; se mantuvo el cultivo durante seis meses, y se hicieron evaluaciones de variables de crecimiento y producción. En cada una de las localidades, se utilizó un invernadero construido según las condiciones climáticas y se midieron variables ambientales a lo

Tabla I. Descripción de tres localidades utilizadas para un experimento de producción de tomate en cultivos protegidos

\begin{tabular}{|c|c|c|c|c|}
\hline Localidad & Altitud $(\mathrm{msnm})$ & $\begin{array}{c}\text { Temperaturas } \\
(\mathrm{min} \text { y max })\end{array}$ & $\begin{array}{c}\text { Precipitación } \\
\text { anual }(\mathrm{mm})\end{array}$ & $\begin{array}{c}\text { Zona de vida según } \\
\text { Holdridge }\end{array}$ \\
\hline $\begin{array}{c}\text { San Blas de Carrillo, } \\
\text { Guanacaste }\end{array}$ & 50 & $17-32$ & 1500 & Bosque seco tropical \\
\hline $\begin{array}{c}\text { Santa Clara de San Carlos, } \\
\text { Zona norte }\end{array}$ & 170 & $15-30$ & 3500 & Bosque húmedo tropical \\
\hline $\begin{array}{c}\text { Zapote de Zarcero, Valle } \\
\text { Central occidental }\end{array}$ & 1500 & $10-22.5$ & 2000 & Bosque húmedo premontano \\
\hline
\end{tabular}

Fuente: http://www.imn.ac.cr/mapa_clima/altas_clima/ 
interno de la estructura. El experimento consistió de tres ensayos idénticos, uno en cada localidad seleccionada, donde se sembraron los hibridos de forma consecutiva, no simultáneamente.

\section{Localización del experimento y periodo de estudio}

Las tres localidades de Costa Rica seleccionadas fueron: San Blas de Carrillo, en la provincia de Guanacaste, Santa Clara de San Carlos, en la provincia de Alajuela y Zapote de Zarcero, en la provincia de Alajuela. Los ensayos se realizaron en diferentes periodos: en San Blas, del 20 de julio al 20 de diciembre del 2007; en Santa Clara, del I de julio al 6 de diciembre del 2008; y, por último, en Zapote de Zarcero, del 2 de marzo al 2 de agosto del 2009. Se escogieron estas localidades por sus diferencias climáticas, de altitud y zonas de vida, según Holdbridge (tabla I).

\section{Descripción de los invernaderos}

Los invernaderos utilizados en el experimento son similares entre sí: todos tienen diseño tipo capilla con monitor o ventana cenital en la parte superior de la estructura y techo en forma de arco; las paredes están cubiertas de malla antiáfidos de nylon color blanco (Arrigoni), con una densidad de $32 \times$ 32 hilos por pulgada lineal; el techo del invernadero está cubierto de plástico transparente con revestimiento para el filtraje de las radiaciones ultravioleta (Lirsa). El piso del invernadero está cubierto con una alfombra plástica semipermeable (Agritela, Arrigoni); se utilizó de color blanco (Agritela Riflex, Arrigoni) en los invernaderos de Santa Clara y Zapote de Zarcero, y de color negro (Agritela Nera, Arrigoni) en el invernadero de San Blas de Carrillo. Se escogieron estos dos tipos de cobertura principalmente porque en las localidades de Santa Clara y Zapote la luminosidad es más baja que en la localidad de San Blas, donde el color blanco de la cobertura favorece la reflectancia de la luz.

Las dimensiones de los invernaderos varían ligeramente según la localidad. Para el invernadero de San Blas de Carrillo las medidas son de 10 metros de ancho por 48 metros de largo, con una apertura cenital de I metro de ancho, una altura de pared de 5 metros y una altura total de 7 metros. El de Santa Clara de San Carlos es de 9 metros de ancho y un largo de 30 metros, tiene una altura de paredes de 4,5 metros pero la altura total es de 7,5 metros y la apertura cenital es de 1,2 metros de ancho. El invernadero de Zapote de Zarcero tiene 3,5 metros de altura de pared y una altura total de 6,5 metros, tiene monitor cenital de 0,8 metros de ancho y es de 10 metros de ancho y 30 metros de longitud.

\section{Material experimental}

El material vegetal utilizado fue tres híbridos de tomate tipo bola (Lycopersicum esculentum M): Híbrido "LSL 449" (Divine Ripe, Israel) con hábito de crecimiento semideterminado; híbrido "Qualyt 2l" (Syngenta Rogers Seeds, Estados Unidos) con hábito de crecimiento determinado; y el híbrido "Sabbia" (Nunhems seeds, Holanda) con hábito de crecimiento indeterminado.

\section{Diseño experimental, tratamientos y análisis estadístico}

El diseño experimental corresponde a un diseño irrestricto al azar; se utiliza un factorial $3 \times 3$, donde interactúan los tres híbridos con tres localidades, para nueve tratamientos resultantes de la interacción; el número de repeticiones por tratamiento fue de cinco, o sea quince unidades experimentales por localidad, y un total de cuarenta y cinco unidades. La unidad experimental consistió de una hilera de diez plantas, con una parcela útil de seis plantas; las cuatro restantes fueron una submuestra utilizada en muestreos destructivos. El modelo del experimento fue el siguiente: $Y_{\mathrm{ij}}=\mu+\mathrm{t}_{\mathrm{i}}+\mathrm{I}_{\mathrm{j}}+\left(\mathrm{t} * \mathrm{w}_{\mathrm{ij}}\right)_{\mathrm{ij}}+\varepsilon_{\mathrm{ij}}$

Donde " $t$ " corresponde a los híbridos de tomate; "'l" a las localidades, "(t*il)" a la interacción híbridos (genotipos) con las localidades; " $\mu$ " es la media general; $y$ " $\varepsilon$ " es el error.

\section{Variables asociadas a la medición del ambiente dentro de los invernaderos}

La temperatura en grados Celsios y la humedad relativa en porcentaje, mínimas y máximas, se tomaron a diario a las 7:30 de la mañana con un higrotermómetro digital (Precision) ubicado en medio del cultivo a una altura de I,5 metros. La luminosidad se midió en luxes diariamente dentro de los invernaderos a las 12:00 m y se utilizó un luxómetro (Luxom Precision).

\section{Análisis estadístico}

Se llevó a cabo un análisis de componentes principales para entender las relaciones entre las variables (Borrego et al, 200 I). Para cada una de las variables 
Tabla 2. Tratamientos factoriales, tres genotipos de tomate con diferente hábito de crecimiento en tres zonas agroecológicas de Costa Rica.

\begin{tabular}{|c|c|c|c|c|}
\hline Interacción localidad $x$ híbrido & Híbrido & Hábito de crecimiento & Localidad & Abreviación \\
\hline Guanacaste $\times$ Determinado & Qualyt 2 & Determinado & Guanacaste & Gte-Det \\
\hline Guanacaste $\times$ Semideterminado & LSL 449 & Semideterminado & Guanacaste & Gte-SemiDet \\
\hline Guanacaste $\times$ Indeterminado & Sabbia & Indeterminado & Guanacaste & Gte-Indet \\
\hline San Carlos $\times$ Determinado & Qualyt 21 & Determinado & San Carlos & SC-Det \\
\hline San Carlos $\times$ Semideterminado & LSL 449 & Semideterminado & San Carlos & SC-SemiDet \\
\hline San Carlos $\times$ Indeterminado & Sabbia & Indeterminado & San Carlos & SC-Indet \\
\hline Zarcero $\times$ Determinado & Qualyt 21 & Determinado & Zarcero & Zar-Det \\
\hline Zarcero $\times$ Semideterminado & LSL 449 & Semideterminado & Zarcero & Zar-Semidet \\
\hline Zarcero $\times$ Indeterminado & Sabbia & Indeterminado & Zarcero & Zar-Indet \\
\hline
\end{tabular}

\section{Variables evaluadas}

Tabla 3. Descripción de las variables evaluadas durante el experimento, para tres híbridos de tomate en tres localidades de Costa Rica

\begin{tabular}{|c|c|c|c|}
\hline Variable & abreviación & Unidad de medición & Frecuencia medición \\
\hline Peso seco total & PST & Gramos/planta & Mensual \\
\hline Peso seco follaje & PSF & Gramos/planta & Mensual \\
\hline Peso seco de tallo & PSTLL & Gramos/planta & Mensual \\
\hline Peso seco de raíz & PSR & Gramos/planta & Mensual \\
\hline Peso seco vegetativo & PSV & Gramos/planta & Mensual \\
\hline Peso seco de frutos & PSFr & Gramos/planta & Mensual \\
\hline Relación vegetativa entre productiva & Veg/Frut & Sin unidades & Mensual \\
\hline Altura de planta & AltPlant & Centímetros & Semanal \\
\hline Grosor de tallo & Grosor & Centímetros & Semanal \\
\hline Hojas por planta & H/PI & Número de hojas & Semanal \\
\hline Racimos por planta & Rc/PI & Número de racimos & Semanal \\
\hline Flores abiertas por planta & Fl/PI & Número de flores & Semanal \\
\hline Frutos por planta & Fr/PI & Número de frutos & Semanal \\
\hline Flores promedio por racimo & Fl/Rac & Número de flores & Semanal \\
\hline Frutos promedio por racimo & Fr/Rac & Número de frutos & Semanal \\
\hline Peso total de frutos por planta & Grs/PI & Gramos & Semanal \\
\hline Peso promedio de frutos & Pfrut & Gramos & Semanal \\
\hline Producción en kilogramos de frutos por & Kg/m2 & Kilogramos & Semanal \\
\hline metro cuadrado & Índice de área foliar $*$ & Sin unidades & Semanal \\
\hline
\end{tabular}

* Método sugerido por Blanco y Folegatti (2003).

seleccionadas, se llevaron a cabo análisis de variancia y pruebas de diferencias entre medias (Tuckey, $p=0,05)$. Por otro lado, se utilizó el programa JMP de SAS Institute versión 8 para el análisis estadístico.

\section{Manejo del cultivo y sistema de siembra}

Los cultivos se manejaron de manera similar para las tres localidades; el sistema de cultivo fue de tipo hidropónico con uso de sustrato inorgánico en contenedores individuales. Se utilizaron plántulas de treinta días de edad al momento del trasplante y 
se les aplicó Trichoderma spp incorporado en el sustrato (Germination Mix, Fafard), y Confidor 70 WG (Imidacloprid) (Bayer) como insecticida preventivo.

Se trasplantó una planta por cada contenedor de diez litros, y el sustrato utilizado fue arena del río Peñas Blancas para los invernaderos de San Carlos y Zarcero, y del río Tempisque para el de Guanacaste. Luego se aplicó riego por goteo con solución hidropónica completa a una frecuencia de nueve riegos diarios, de dos a cinco minutos cada uno programados con un temporizador (Nelson timer). A las plantas se les dejó dos ramas o guías principales, que se tutoraron con dos cuerdas por planta, y se podaron los brotes laterales.

Los contenedores se colocaron en hileras de 9 metros de largo, con una distancia entre ellos de 0,4 metros, y entre hileras de 1,5 metros, para una densidad de 16.665 plantas por hectárea.

\section{Manejo de plagas y enfermedades}

Las plagas y enfermedades se manejaron mediante un control biológico y químico cuando fue necesario, se usaron, de manera preventiva, aplicaciones de hongos antagonistas y entomopatógenos, como Trichoderma spp, Peaecelomyces spp y Metarhizium spp, y los insecticidas Imidacloprid (Confidor 70 WG) y Abamectina (Vertimec), además de los fungicidas Metalaxil (Ridomil MZ) y Sportak (Bayer).

\section{Resultados y discusión}

Se llevó a cabo un análisis de correlación entre las variables, del que se obtuvo como resultado un alto nivel entre muchas de ellas; en la figura I se presenta un conglomerado de las correlaciones obtenidas, donde se puede observar que muchas de las variables presentan alta correlación, lo que forma grupos o conglomerados que corresponden a las variables que están altamente correlacionadas.

Con base en la matriz de correlaciones, se realizó un análisis de componentes principales (Rodríguez, 2009; y Rodríguez, 2009) donde las variables se estandarizan, de manera que la varianza total en los resultados del experimento sea igual al número total de variables.

El análisis de componentes principales tiene como objetivo reducir la dimensión de la variabilidad al perder la menor cantidad posible, cada componente

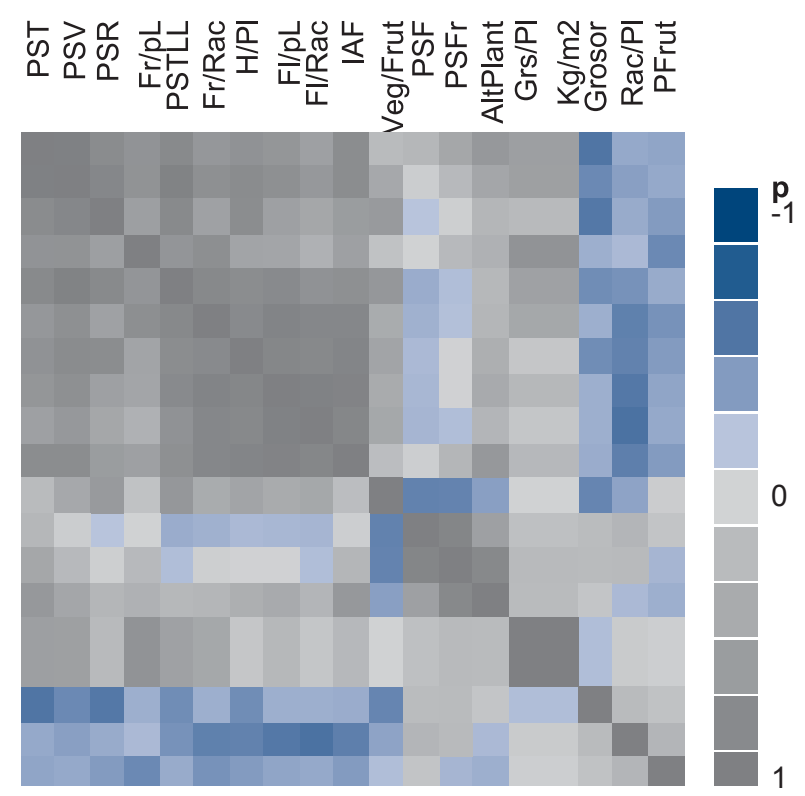

Figura I. Cluster o conglomerado de correlaciones de diecinueve variables evaluadas para tres hábitos de crecimiento de tomate en tres localidades bajo sistema de cultivo protegido.

es una combinación lineal de las variables originales estandarizadas (Rodríguez, 2009).

Cada uno de los componentes principales es independiente ya que no están correlacionados entre sí y van a poseer un valor, el cual corresponde a la varianza que este explica e indica el nivel de contribución que tiene a la variabilidad total; este valor se denomina "eigenvalor". En la tabla 4 se muestran esos valores para ocho componentes principales resultantes del análisis; observese que siempre el primer componente es el que explica la mayor variabilidad, el segundo explica la que no se explicó en el primero, de manera que el eingenvalor va disminuyendo sucesivamente para el resto de los componentes; también se muestra el porcentaje acumulado de la variabilidad explicada para cada uno.

Según los resultados, los dos primeros componentes explican el 70,052\% de la variabilidad total y consideran el principio de "parsinomía", por lo que se pueden seleccionar para nuestro análisis ya que explican la mayor variabilidad posible con el mínimo posible de componentes (Rodríguez, 2009). 
Tabla 4. Proporción de la varianza explicada por ocho componentes principales para diecinueve variables de respuesta de tres hábitos de crecimiento de tomate en tres localidades.

\begin{tabular}{|c|c|c|c|}
$\begin{array}{c}\text { Componente } \\
\text { principal }\end{array}$ & Eigenvalue & Porcentaje & $\begin{array}{c}\text { Porcentaje } \\
\text { acumulado }\end{array}$ \\
\hline 1 & 9,4734 & 49,860 & 49,860 \\
\hline 2 & 3,8364 & 20,192 & 70,052 \\
3 & 2,3721 & 12,485 & 82,537 \\
4 & 1,5596 & 8,209 & 90,745 \\
5 & 1,1642 & 6,127 & 96,873 \\
6 & 0,3576 & 1,882 & 98,755 \\
7 & 0,1674 & 0,881 & 99,636 \\
8 & 0,0692 & 0,364 & 100,000 \\
\hline
\end{tabular}

Cada componente reúne variables altamente correlacionadas, y es posible elegir las variables representativas de cada componente si se toma en cuenta que su valor sea alto en el componente elegido y bajo en los otros.

En cada uno de los componentes principales, las variables que los componen tienen un peso o magnitud dentro de cada uno, llamado coeficiente, que define, en cierta medida, al componente y le dan su significado; ese valor o magnitud es denominado "eigenvector".

Según los resultados presentes en la tabla 5, las siete variables con mayor eigenvector asociado al componente principal I (CPI) son: peso seco de tallos (PSTLL), peso seco vegetativo (PSV), número de hojas por planta $(\mathrm{H} / \mathrm{PI})$, índice de área foliar (IAF), número de frutos por racimo (Fr/Rac), flores por planta (FI/PI) y peso seco total (PST); estas fueron seleccionadas como representativas del CPI no solo por el valor de eigenvector, sino también por el siginificado que estas tienen desde el punto de vista de crecimiento de la planta, ya que representan medidas directas del crecimiento (Salisbury y Ross, 1994).

Tanto los pesos secos de las diferentes fracciones y su totalidad, como el número de órganos, reflejan, a nivel fisiológico, los cambios bioquímicos que se manifiestan, como un aumento irreversible en el tamaño de los organismos (Barraza, Fischer y Cardona 2004). Este componente principal I (CPI) podría denominarse como el componente que explica el crecimiento del cultivo o componente del crecimiento del cultivo, debido a que representa el
Tabla 5. Eigenvectores para las variables de repuesta de los componentes I y 2 para tres genotipos de tomate en tres localidades.

\begin{tabular}{|c|c|c|}
\hline Variable & Componente I & Componente 2 \\
\hline PST & $0,2844 *^{*}$ & 0,19723 \\
\hline PSF & $-0,04261$ & $0,43273^{*}$ \\
\hline PSTLL & $0,30576 *$ & $-0,06593$ \\
\hline PSR & 0,26259 & $-0,06366$ \\
\hline PSV & $0,3048 *^{*}$ & 0,08361 \\
\hline PSFr & 0,02682 & $0,47512^{*}$ \\
\hline Veg/Frut & 0,15573 & $-0,40254$ \\
\hline AltPlant & 0,13600 & $0,36813^{*}$ \\
\hline Grosor & $-0,18267$ & 0,17286 \\
\hline H/PI & $0,29238^{*}$ & $-0,08929$ \\
\hline Rac/PI & $-0,22010$ & 0,18491 \\
\hline FI/pl & $0,29020 *$ & $-0,05528$ \\
\hline Fr/pl & 0,23387 & 0,16011 \\
\hline Fl/Rac & 0,26596 & $-0,12197$ \\
\hline Fr/Rac & $0,29496 *$ & $-0,03473$ \\
\hline Grs/PI & 0,14340 & $0,23736 *$ \\
\hline PFrut & $-0,16751$ & $-0,03026$ \\
\hline Kg/m2 & 0,14340 & $0,23736 *$ \\
\hline IAF & $0,29207 *$ & 0,06888 \\
\hline
\end{tabular}

* Valores con mayor peso seleccionados para describir al componente.

aumento en biomasa y número de órganos en la planta.

En el componente 2 (CP2), las cinco variables de mayor magnitud son: peso seco de frutos (PSFr), peso seco del follaje (PSF), altura de planta (AltPlant), gramos de frutos cosechados por planta ( $\mathrm{Grs} / \mathrm{Pl}$ ) y kilogramos de frutos por metro cuadrado $(\mathrm{Kg} / \mathrm{m} 2)$. Estas últimas dos variables están asociadas al rendimiento, así como el cúmulo de materia seca a nivel de frutos (PSFr) con mayor magnitud en este componente; también tenemos dos variables importantes que son peso seco de follaje (PSF) y altura de planta (AltPlant), relacionadas con crecimiento y desarrollo del cultivo, de las que todo el peso seco del follaje (PSF) es la más relevante.

Existe una relación denominada "fuente/sumidero" que hace referencia a la traslocación de asimilados fotosintéticos de la fracción vegetativa (fuente) de la planta hacia la fracción reproductiva (sumidero), en este caso la fracción vegetativa está representada por el PSF y la reproductiva por el PSFr. Esta relación es muy directa o paralela entre el tamaño de la fuente y el tamaño alcanzado por el sumidero, que posee 
habilidad competitiva por atraer asimilados fotosintéticos del follaje (Dogliotti, 2002; Villalobos, 200 I).

El componente 2 (CP2) tiene variables asociadas a la parte reproductiva, como lo son las relacionadas a los frutos y al rendimiento, además del crecimiento de la planta, por lo que sería un componente con un significado mixto, con un aporte importante de las variables productivas y vegetativas asociadas al crecimiento. Se puede afirmar que es un componente que representa el rendimiento, ya que se asocian variables que lo explican en función de la relación fuente/sumidero y la producción por planta, y da significado al efecto que tiene el peso seco del follaje en el cúmulo de biomasa por parte de los frutos.

Si se consideran las variables con mayor peso en estos dos primeros componentes principales, queda en evidencia la importancia que tienen las variables asociadas al cúmulo de materia seca y a la productividad del cultivo del tomate dentro de los resultados de este experimento.

La variable peso seco total (PST) es una variable compuesta por la suma de las fracciones de materia seca de la planta. Heuvelink (1996) estudió la parti- ción de la materia seca del cultivo de tomate a través de un modelo de simulación que representaba la relación entre la materia seca reproductiva con la vegetativa. En nuestro caso, la variable que representa el cúmulo de materia seca de los frutos (PSFr) es de gran peso en el componente 2, mientras que las de cúmulo de materia seca vegetativa, peso seco vegetativo (PSV) y peso seco de tallos (PSTLL) tienen mayor importancia en el componente I; esta partición de la materia seca es importante para demostrar la eficiencia de la planta en distribuir sus asimilados producto de la fotosíntesis, y que ha de manifestarse al final en la productividad del cultivo (Dogliotti, 2002).

El peso seco del follaje (PSF) resultó ser más importante en el componente principal 2 que en el componente I, donde tienen mayor peso las variables de materia seca vegetativa (PSTLL y PSV); al ser las hojas los principales órganos encargados de la fotosíntesis y de la acumulación de sus productos, y al conocer el vínculo fuente/sumidero, podemos entender la importancia de la relación del PSF con el PSFr, que es una relación directa con la productividad final del cultivo. Heuvelink (1995) encontró

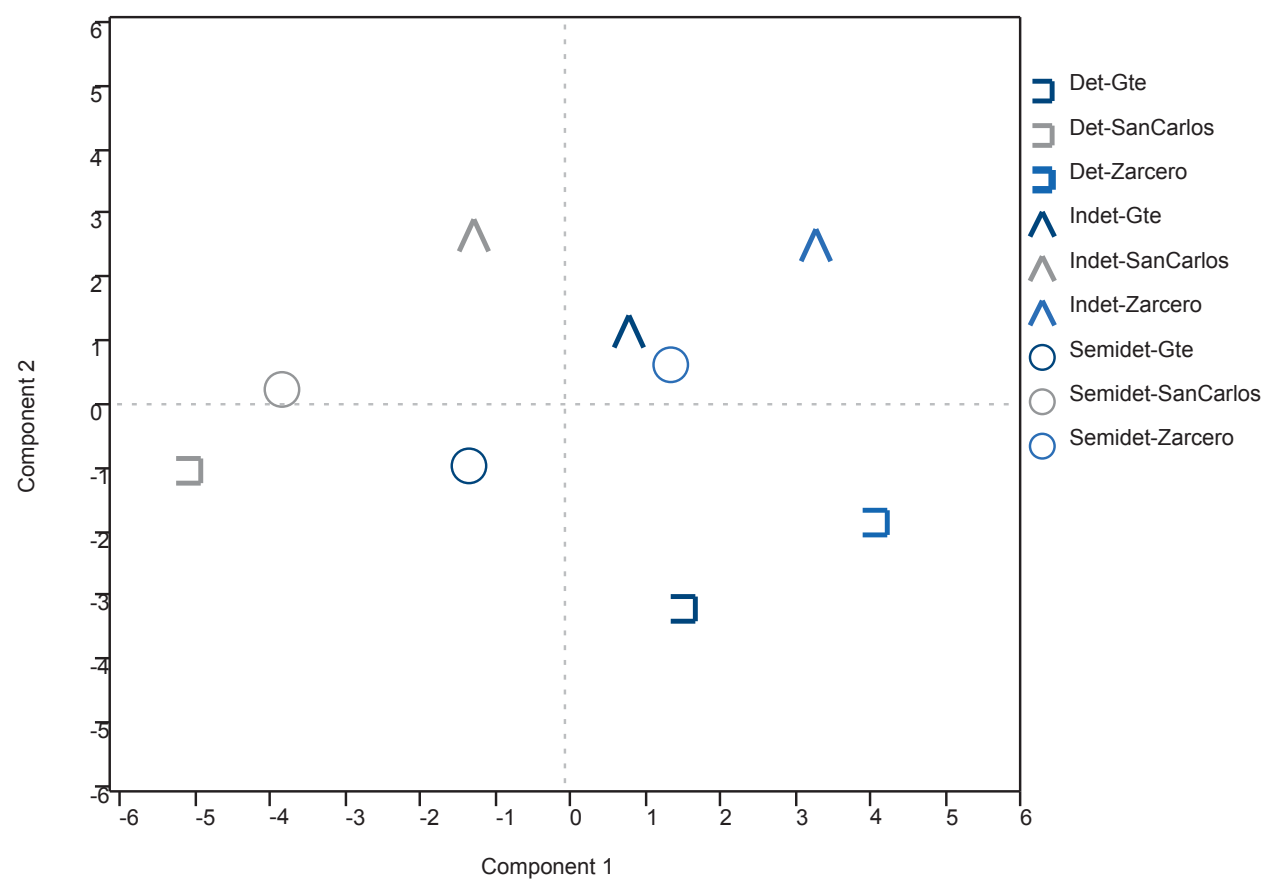

Figura 2. Relación entre los componentes I (49,68\% variabilidad) y 2 (20,2 \% variabilidad) para tres genotipos de tomate en tres localidades bajo sistema de cultivo protegido. 
que del total de materia seca acumulada por planta de tomate bajo invernadero, de un 54\% a un 60\% se distribuía a los frutos, y que más del $50 \%$ de esta materia proviene de las hojas, en comparación con la proveniente de los tallos o de otro órgano de la planta. La importancia de la variable "Altura de planta” en el componente 2 podría explicarse en términos de la acción del sumidero, ya que, conforme se da la traslocación de asimilados fotosintéticos de la parte vegetativa hacia los frutos, el crecimiento se detiene, sobretodo en plantas de ciclo anual como el tomate, donde la cosecha se concentra al final de su ciclo de vida (Villalobos, 200 I; Castilla, 2004).

Otra consideración importante es que las variables representativas del componente I (CPI) se refieren a estructuras de la planta con crecimiento de tipo indeterminado, que dependen de la acción de un meristemo con potencial de continuar en división celular (Salisbury y Ross, 1994), como es el caso del tallo de las plantas; por otra parte, en el componente 2 (CP2), sus variables representativas corresponden a estructuras de la planta con un crecimiento determinado, o sea, que alcanzan un cierto tamaño y luego senecen y mueren, como es el caso de las hojas y frutos, esto hace una diferencia entre ambos componentes, que ayuda a caracterizarlos como los componentes que explican el crecimiento y el rendimiento, respectivamente.

Borrego et al (200l) encontraron, a través del uso de análisis de componentes principales, que en el cultivo de tomate en invernadero, uno de los componentes se asociaba a las características del rendi- miento y otro a variables fisiológicas de crecimiento, similares a los resultados descritos.

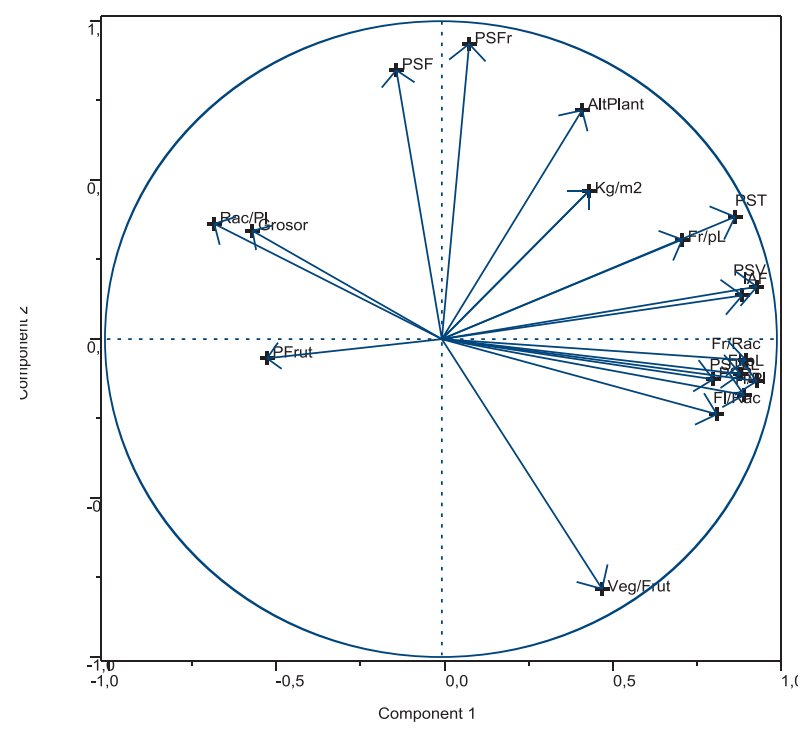

Figura 3. "Loadings" o "factores de carga" de diecinueve variables para los componentes I y 2 evaluados para tres hábitos de crecimiento de tomate en tres localidades bajo sistema de cultivo protegido.

En la figura 3 se presentan las posiciones de cada variable respecto a los componentes I y 2; los puntos de cada variable son la intersección de los loadings (factor de carga) de cada una de ellas para cada componente. Como los componentes se relacionan entre sí de forma perpendicular u ortogonal, cada punto que representa una variable genera un vector con una magnitud que refleja el peso de la variable y la dirección que indica el componente al cual es afín.

Tabla 6. Significancia estadística para las variables con mayor peso en los componentes principales I y 2 según el análisis de varianza a $p=0,05$

Componente I

Componente 2

\begin{tabular}{|c|c|c|c|c|c|c|c|c|c|c|c|}
\hline Fte. de Variación & PSTLL & PST & PSV & $H / P I$ & $\begin{array}{l}\mathrm{Frl} \\
\mathrm{Rac}\end{array}$ & $I A F$ & PSF & PSFr & Grs/PI & $\begin{array}{l}\mathrm{Kg} / \\
\mathrm{m} 2\end{array}$ & AltPlant \\
\hline $\begin{array}{l}\text { Hábito de } \\
\text { crecimiento }\end{array}$ & **⿻丷木 & **⿻丷木 & **⿻丷木 & *** & NS & NS & **⿻ & 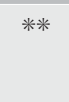 & **⿻丷木 & ** & *** \\
\hline Localidad & **⿻丷木 & NS & *** & *** & *** & *** & NS & NS & **⿻丷木 & **** & *** \\
\hline $\begin{array}{l}\text { Hábito de } \\
\text { crecimiento X } \\
\text { Localidad }\end{array}$ & **** & ***** & **** & ***** & NS & NS & NS & **** & ***** & **** & **** \\
\hline
\end{tabular}

*** $y$ **** : presenta diferencias significativas.

NS : no presenta diferencias significativas. 
Tabla 7. Diferencias entre medias (Tuckey a $\mathrm{p}=0,05$ ) para las variables con mayor peso en los componentes principales I y 2 según los tratamientos

\begin{tabular}{|c|c|c|c|c|c|c|c|c|c|}
\hline \multirow[b]{2}{*}{ Tratamientos } & \multicolumn{4}{|c|}{ Componente I } & \multicolumn{5}{|c|}{ Componente 2} \\
\hline & $\begin{array}{c}\text { PSTLL } \\
\text { (grs) }\end{array}$ & $\begin{array}{l}\text { PST } \\
\text { (grs) }\end{array}$ & $\begin{array}{l}\text { PSV } \\
\text { (grs) }\end{array}$ & $H / P I$ & $\begin{array}{l}\text { PSF } \\
\text { (grs) }\end{array}$ & $\begin{array}{l}\text { PSFr } \\
\text { (grs) }\end{array}$ & Grs/PI & $\mathrm{Kg} / \mathrm{m} 2$ & $\begin{array}{c}\text { AltPlant } \\
\text { (cms) }\end{array}$ \\
\hline Gte-Det & $246.8 a$ & $441.6 b c$ & 423.2ab & $34 b c$ & | |3.2d & $38.46 c$ & $5970.6 c d$ & $7.46 \mathrm{~cd}$ & $164.8 \mathrm{e}$ \\
\hline Gte-SemiDet & $119.3 c$ & $339.7 d$ & $298.42 c$ & $31.5 c$ & $160.84 \mathrm{bc}$ & $41.26 b c$ & $5827.2 d$ & $7.28 d$ & 176.2de \\
\hline Gte-Indet & $136.16 c$ & $470.5 b c$ & $385.72 b$ & $38 b$ & $202.4 \mathrm{a}$ & $84.8 \mathrm{a}$ & $4649.7 e$ & $5.81 \mathrm{e}$ & $270 b$ \\
\hline SC-Det & $44.02 d$ & $221.7 e$ & 185.22d & $22.6 \mathrm{e}$ & I26.06cd & $36.5 c$ & $4600.5 e$ & $5.75 e$ & 177de \\
\hline SC-SemiDet & $105.12 c$ & $338.9 d$ & $296.62 c$ & $25 \mathrm{de}$ & I $72.44 \mathrm{c}$ & $42.26 b c$ & $6964.8 \mathrm{bc}$ & $8.7 \mathrm{lbc}$ & 183.6de \\
\hline SC-Indet & $136.16 c$ & $470.5 b c$ & $385.72 b$ & $27 d$ & $202.4 c$ & $84.8 a$ & $7725.6 a b$ & $9.66 a b$ & $207.4 c$ \\
\hline Zar-Det & $269.8 a$ & $489.2 b$ & $463.2 \mathrm{a}$ & $43.4 \mathrm{a}$ & $121 d$ & $26 d$ & $7005.3 b c$ & $8.76 b c$ & $187.8 \mathrm{~cd}$ \\
\hline Zar-Semidet & $196.6 b$ & $429.6 c$ & $382.2 b$ & $31.6 c$ & $160 b c$ & $47.4 b$ & $8221.2 \mathrm{a}$ & $10.28 \mathrm{a}$ & $196.6 \mathrm{~cd}$ \\
\hline Zar-Indet & $256.6 a$ & $558.8^{a}$ & $476 a$ & $35 b c$ & 166.4ab & $82.8 a$ & $8302.5^{a}$ & $10.38^{a}$ & $297.2^{\mathrm{a}}$ \\
\hline
\end{tabular}

*Letras iguales no muestran diferencias significativas entre tratamientos para cada variable.

La suma de cuadrados de los "loadings" o "pesos" de un componente es la cantidad de varianza que explica el componente, o sea su eigenvalor, y existe una relación directa entre estos y los eigenvectores para cada variable, dada por la ecuación:

factor de carga (loading) = eigenvector $*$ eigenvalor ${ }^{1 / 2}$ (Vicente, s.f., y Rodríguez, 2009)

Algunas variables, como el PSFr, poseen un loading alto para CP2, y su vector correspondiente en la gráfica indica su magnitud y su afinidad hacia ese componente, ya que es casi paralelo a este; igualmente, para la variable PSTLL, que corresponde a la variable con mayor peso para $\mathrm{CPI}$, este vector resultante se orienta prácticamente paralelo al eje que representa al componente.

Las variables PSFr y PSTLL son las significativas de los componentes I y 2 por tener su máximo eigenvector, y por representar, desde el punto de vista fisiológico, dos modos de crecimiento de la planta, como se mencionó anteriormente.

La interacción de tres genotipos en tres localidades (tratamientos) también influye en la variabilidad del sistema y tiene su peso dentro de los componentes principales, ese peso o magnitud (eigenvector) nos va a indicar cómo se comporta cada genotipo según el ambiente (localidad) y viceversa, y explican esa relación en función de los componentes principales I y 2. La posición de los tratamientos respecto a estos componentes principales se muestran en la figura 2 .

Los tratamientos con mayor peso en el componente I son los genotipos evaluados en la localidad de Zarcero, y de menor peso los evaluados en San Carlos, mientras que los genotipos evaluados en Guanacaste muestran un comportamiento intermedio.

Se puede apreciar también cierto nivel de agrupamiento por localidades con valores similares para el componente I, y un comportamiento diferente

Tabla 8. Comportamiento de variables climáticas internas de los invernaderos, para tres localidades de Costa Rica durante el periodo de estudio

\begin{tabular}{|c|c|c|c|c|c|c|}
\hline Localidad & $\begin{array}{c}\text { Temperatura } \\
\text { promedio } \\
\text { máxima }\end{array}$ & $\begin{array}{c}\text { Temperatura } \\
\text { promedio } \\
\text { mínima }\end{array}$ & $\begin{array}{c}\text { Humedad } \\
\text { relativa } \\
\text { promedio } \\
\text { máxima }\end{array}$ & $\begin{array}{c}\text { Humedad } \\
\text { relativa } \\
\text { promedio } \\
\text { mínima }\end{array}$ & $\begin{array}{c}\text { Integral } \\
\text { térmica } \\
\text { (grados día) }\end{array}$ & $\begin{array}{c}\text { Luz promedio } \\
\text { (luxes) }\end{array}$ \\
\hline Guanacaste & $37,28 \mathrm{C}$ & $22,64 \mathrm{C}$ & $87,48 \%$ & $48,02 \%$ & 9573 & 41888,89 \\
San Carlos & $38,09 \mathrm{C}$ & $21,71 \mathrm{C}$ & $88,17 \%$ & $40,66 \%$ & 9502 & 30322,22 \\
Zarcero & $35,90 \mathrm{C}$ & I2,34 C & $87,48 \%$ & $48,02 \%$ & 7719 & 24481,11 \\
\hline
\end{tabular}


entre genotipos iguales según el ambiente (localidades) donde se desarrollaron. Paez, Paz y López (2000), encontraron diferencias en crecimiento y en distribución de la materia seca del tomate cultivar "Río Grande" cuando se cultivó en condiciones ambientales diferentes de luz y sombra; según nuestros resultados, un mismo genotipo presenta valores diferentes para al componente I, el cual representa el crecimiento.

El distanciamiento entre genotipos para el componente I se da más en el genotipo de hábito determinado que en el de hábito indeterminado, con cierto grado de agrupamiento; el hábito de crecimiento indeterminado corresponde a una planta que mantiene un crecimiento sostenido durante su ciclo de vida y su producción se presenta escalonadamente, no concentrada como en los genotipos determinados, los cuales detienen su crecimiento en ese momento (Benton, 2008, y Castellanos, 2009). Este comportamiento natural de las plantas de tomate indeterminadas podría explicar el poco distanciamiento que tiene este genotipo en las diferentes localidades respecto al genotipo de hábito determinado, que muestra un distanciamiento amplio según la localidad para el componente I (CPI). Santiago, Mendoza y Borrego (1998) evaluaron genotipos de tomate de tipo determinado en invernadero y obtuvieron diferencias entre ellos en productividad y fotosíntesis, pese a tener el mismo hábito de crecimiento.

Para el caso del componente 2 (CP2), que representa el rendimiento, se puede observar también cierto nivel de agrupamiento por genotipos, lo que muestra mayores valores el genotipo indeterminado con valores similares independientemente de la localidad; por otra parte el genotipo determinado presentó menores valores, mientras el semideterminado, valores intermedios; el componente 2 (CP2) es el asociado al rendimiento, y es el hábito indeterminado el que más aporta a este componente, lo cual coincide con un mayor PSFr en comparación con los otros hábitos de crecimiento (tablas 6 y 7 ).

Para el componente 2 (CP2), la variable AltPlant tiene un alto peso y es una característica asociada al hábito de crecimiento; el hábito indeterminado es el que muestra valores altos, y es normal que este genotipo sea más alto que los determinados o semideterminados (tabla 7). La altura de la planta asociada al hábito de crecimiento queda en evidencia en la distribución y agrupamiento de las interacciones en el componente 2 (CP2), mostradas en la figura 2

Los genotipos mostraron un comportamiento diferenciado según localidad para el componente I (CPI), y para el componente 2 (CP2) las diferencias se mostraron más a nivel de localidades que de genotipos.

Los resultados de las variables más representativas de los componentes y su significancia se muestran en las tablas 6 y 7. Para muchas variables resultó significativa la interacción del hábito de crecimiento con la localidad, al igual que en la figura I, donde se mostró el distanciamiento entre tratamientos.

Las variables de mayor interés para cualquier productor son las relacionadas con el rendimiento (componente principal 2); en la tabla 7 se muestran las productividades para los diferentes tratamientos, que fueron mayores en los genotipos sembrados en la zona de Zarcero, con producciones por planta cercanas a los $10 \mathrm{Kg} / \mathrm{m}^{2}$ de fruta fresca. Si se compara este dato con los reportados por Castilla (2005), de $18 \mathrm{Kg} / \mathrm{m}^{2}$ para España y de $58 \mathrm{Kg} / \mathrm{m}^{2}$ para Holanda, estaríamos muy por debajo de ellos, sin embargo esto no significa que todos los genotipos con los hábitos de crecimiento estudiados, vayan a presentar productividades similares, ya que pueden tener adaptaciones distintas según la localidad y el sistema de cultivo protegido en el trópico.

El genotipo de crecimiento indeterminado fue uno de los que presentó mejores producciones, lo que coincide con lo recomendado por muchos autores para uso en invernadero (Gil, Sánchez del Castillo y Miranda, 2003; Rodríguez, Muñoz y Alcorta, 2006), sin embargo, en San Carlos, tanto el genotipo determinado como el semideterminado mostraron producciones similares al indeterminado; mientras que en Guanacaste, el determinado tuvo la mayor producción, lo cual pudo estar influenciado por el ambiente, lo que sugiere que en el trópico podrían utilizarse genotipos determinados en invernadero, contrario a lo recomendado.

El manejo del cultivo también puede condicionar su productividad, así como las podas y deshijas, llevadas a cabo por igual para todos los genotipos, puesto que varían la relación fuente/sumidero, ya que al eliminar los brotes laterales se estimula el crecimiento de los frutos ante la falta de competencia por los asimilados fotosintéticos (Villalobos, 200 I; Castilla, 2004). 
Pese a que se cultivó en un sistema de tipo hidropónico y que los invernaderos son estructuralmente similares, se presentaron diferencias climáticas en el interior según la localidad, como se muestra en la tabla 8; por ejemplo, la temperatura mínima promedio fue más baja en el invernadero de Zarcero, y, como consecuencia se obtuvo una integral térmica menor, aunque las humedades relativas son bastante similares, aunque sí se ven diferencias en el promedio de luminosidad entre las localidades, siendo menor en Zarcero; en las localidades de San Carlos y Guanacaste se presentaron temperaturas altas, que pudieron haber afectado el crecimiento de tallos y estructuras vegetativas de las plantas (Gil, 2003).

A pesar de que el experimento se realizó en invernaderos, se encontraron fuertes interacciones entre genotipos y ambientes, lo que significa que los genotipos tienen una adaptación muy específica a las diferentes zonas agroecológicas o localidades.Borrego et al (200 I) y Santiago et al (1998) encontraron diferencias importantes en producción entre los genotipos evaluados bajo invernadero, que representa una clara adaptación de algunos de estos a sus condiciones de evaluación.

Las tres localidades donde se llevaron a cabo los ensayos pertenecen a zonas de vida distintas, según Holdbridge. Las diferencias mostradas entre tratamientos para los componentes seleccionados también se mostraron para sus variables representativas; todas esas diferencias mostradas entre genotipos, dejan claro la influencia del ambiente en la manifestación de ciertas variables asociadas al crecimiento y a la producción de los híbridos de tomate.

\section{Conclusiones}

Bajo las condiciones en que se llevó a cabo el experimento, y con base en los resultados, se concluyó lo siguiente:

- El análisis de componentes principales mostró la importancia de las variables asociadas al crecimiento y al rendimiento, y destacaron las obtenidas a partir de la determinación de la materia seca.

Los componentes I y 2 representaron el crecimiento y la productividad, y sus variables representativas fueron "Peso seco de tallos" y "Peso seco de frutos" respectivamente.
- Se encontraron fuertes efectos de ambientes y genotipos, además de sus interacciones, lo que indica una adaptación específica de cada genotipo para las localidades seleccionadas pese a tener invernaderos similares.

- Los niveles de producción máximos alcanzados en algunos de los tratamientos son muy inferiores a los reportados por la literatura para el cultivo de tomate en sistemas protegidos en otras latitudes.

- En la localidad de Guanacaste, el genotipo de hábito determinado mostró mayor producción que el hábito indeterminado.

\section{Recomendaciones}

Se pueden realizar experimentos con base en los resultados con el fin de ampliar el rango de genotipos determinados para establecer el nivel de variancia entre ellos en un mismo ambiente.

Para la localidad de Guanacaste sería conveniente verificar el resultado de mayor productividad del genotipo determinado sobre el indeterminado.

Se debe considerar, en experimentos futuros, variables asociadas para indicar la sostenibilidad del sistema de producción.

\section{Bibliografía}

Barraza, F;; Fischer, G. \& Cardona, C. (2004). Estudio del proceso de crecimiento del cultivo del tomate (Lycopersicon esculentum Mill.) en el valle de Sinú Medio, Colombia. Agronomía colombiana, 22 (I), 81-90.

Benton, J. (2008). Tomato Plant Culture in the Field, Greenhouse, and Home Garden. (ed. 2) (p. 397). Florida: CRC Press.

Blanco, F. \& Folegatti, M. (2003). A new method for estimating the leaf area index of cucumber and tomato plants. Horticultura Brasileira, 2 I (4).

Borrego, F.; López,A.; Fernández,.:; Murillo, M.; Rodríguez, S.; Reyes,A. \& Martínez,J. (200 I). Evaluación agronómica de tomate (Lycopersicon esculentum Mill.) en invernadero. Agronomía Mesoamericana, 2 I (I), 49-56.

Castellanos, J. (2009). Manual de producción de tomate en invernadero (p. 460). Celaya: Intagri S.C.

Castilla, N. (2004). Invernaderos de plástico: tecnología y manejo (p. 457). Madrid: Mundi Prensa. 
Consejo Nacional de Producción. (2008). Tomate Costa Rica, Subgerencia de desarrollo agropecuario, dirección de mercadeo y agroindustria. Boletín No I. Recuperado de http://mercanet.cnp.go.cr.

Dogliotti, S. (2002). Bases fisiológicas del crecimiento y desarrollo del cultivo del tomate (Lycopersicon esculentum Mill.) [Material de apoyo]. Uruguay: Universidad de la República: Curso de Fisiología de los Cultivos.

Gil, L:; Sánchez del Castillo, F. \& Miranda, I. (2003). Producción de jitomate en hidroponía bajo invernadero (p. 90). Chapingo: Serie de publicaciones Agribot.

Heuvelink, E. (1995). Growth, Development and Yield of a Tomato Crop: Periodic Destructive Measurements in a Greenhouse. Scientia horticulturae, 61, 77-99.

Paez, A.; Paz, V. \& López, J. (2000). Crecimiento y respuestas fisiológicas de plantas de tomate cv. Río Grande en la época mayo-julio. Efecto sombreado. Revista de la Facultad de Agronomía Luz, 17, 173- 184.

Rodríguez, J. (2009). Estadística Análisis de componentes principales [Notas de clase]. Viña del Mar, Chile: Universidad de Viña del Mar: Sociología.
Rodríguez, H.; Muñoz, S. \& Alcorta, E. (2006). El Tomate Rojo: Sistema Hidropónico. México D.F.:Trillas.

Rodríguez, O. (2009). Análisis en Componentes Principales. Recuperado de http://oldemarrodriguez.com/yahoo_ site_admin/assets/docs/cap2.23380802.pdf.

Salisbury, F. \& Ross, C. (1994). Fisiología vegetal (p. 759). México D.F.: Iberoamérica.

Santiago, J.; Mendoza, M. \& Borrego, F. (1998). Evaluación de tomate (Lycopersicon esculentum Mill.) en invernadero: criterios fenológicos y fisiológicos. Agronomía Mesoamericana, 9 (I), 59-65.

Vicente, J. (s.f.). Análisis de componentes principales [Notas de curso]. Salamanca, España: Universidad de Salamanca, Departamento de Estadística.

Villalobos, E. (200I). Fisiología de la producción de los cultivos tropicales, procesos fisiológicos básicos. Fascículo I. San José: Editorial de la Universidad de Costa Rica.

Witter, S. \& Castilla, N. (1995). Protected Cultivation of Horticultural Crops Worldwide. HortTechnology, 5 (I), 6-22. 